\title{
Basal phenotype of ductal carcinoma in situ: recognition and immunohistologic profile
}

\author{
David J Dabbs, Mamatha Chivukula, Gloria Carter and Rohit Bhargava \\ Department of Pathology, Magee-Women's Hospital of UPMC, Pittsburgh, PA, USA
}

\begin{abstract}
The basal phenotype of breast carcinoma was demonstrated from a study of gene expression profiles, which demonstrated five carcinoma phenotypes with differing immunohistologic profiles and outcomes. The basal phenotype, so-named because of an immunohistologic profile that is similar to myoepithelial cells of the breast, has poor outcomes. While the invasive basal phenotype has been described, there is a paucity of literature regarding the existence or recognition of a precursor lesion. We searched our CoPath database for breast carcinomas in the age group of 37 years or less, and this yielded 98 cases from the years 2001 to April 2006. Pathology reports were screened for those cases that were negative for estrogen and progesterone receptors and HER-2/neu (triple negative). A total of 16 cases (16/98, 16\%) fulfilled these criteria. Histology was reviewed and immunostains were performed for Cytokeratins 14, 17, and 5/6, vimentin, EGFR, c-kit, smooth muscle actin and p63. All 16 cases had a high-grade invasive ductal carcinoma, Nottingham score 9/9, with geographic necrosis, good circumscription and lymphoid infiltrates. Of the 16 cases, 13 exhibited at least one area of ductal carcinoma in situ (DCIS). The DCIS types were solid, flat or micropapillary, high nuclear grade, with comedonecrosis and invariably associated with intense lymphoid inflammatory cell infiltration. Of 16 invasive cases, $14(88 \%)$ were positive for CK14, CK17, CK5/6 and EGFR; 94\% were vimentin positive, while half or less of cases were positive for smooth muscle actin, c-kit or p63. All of the DCIS components demonstrated the same immunohistologic profile as the invasive component. A DCIS component of solid, flat or micropapillary type exists in the basal phenotype of breast carcinoma, and it demonstrates the same immunophenotype as the invasive carcinoma, typically positive for CK5/6, CK14, CK17, vimentin and EGFR, but negative for ER/PR and HER-2/neu.

Modern Pathology (2006) 19, 1506-1511. doi:10.1038/modpathol.3800678; published online 25 August 2006
\end{abstract}

Keywords: basal-like; breast carcinoma; immunohistochemistry

The evolution of breast cancer remains poorly understood, although recent gene expression profiling studies suggest the existence of five basic phenotypes, the normal breast type, luminal A, luminal B, Her2 type and basal type. ${ }^{1-4}$ As the recognition of the different epithelial phenotypes become more widespread, tailored therapeutic strategies may become more important.

The mammary epithelium is composed fundamentally of two cell types, basal (myoepithelial) and luminal $(A \& B)$ epithelium. Each cell type is capable of being represented as a phenotype of invasive carcinoma, with a distinct histology, as well as a distinct receptor expression. The focus on the subset of 'basal-like' breast carcinomas, a previously poorly

Correspondence: Dr DJ Dabbs, MD, Department of Pathology, Magee-Women's Hospital of UPMC, 300 Halket St, Pittsburgh, PA 15213, USA.

E-mail: ddabbs@upmc.edu

Received 8 June 2006; accepted 31 July 2006; published online 25 August 2006 characterized group, has generated great interest in recent years because of its distinctive high-grade histologic presentation.

Basal-like breast carcinomas express antigens that are typically expressed on the normal basal or myoepithelial cells of the mammary epithelium, and demonstrate histological features of solid architecture, pushing borders, prominent lymphocytic infiltration, geographic necrosis, high Notthingham score and poor nuclear grade. ${ }^{5}$ The characteristic immunohistologic phenotype of these tumors is the 'triple negative' receptors of estrogen, progesterone and HER-2/neu, while expressing keratins CK5/6, CK14, CK17, vimentin and HER-1. ${ }^{5-11}$

While the invasive basal-like neoplasms are reasonably well characterized morphologically and immunohistochemically, there is little data about the precursor lesion(s) for the entity.

In this study, we survey a group of patients who have classically defined basal-like breast tumors in order to investigate, by morphology and immunohistochemistry, the existence and possible kinship of precursor lesion(s). 


\section{Materials and methods}

We searched our CoPath anatomic laboratory database for breast carcinomas occurring in patients of age 37 years or less, and this yielded 98 cases from the time period November 2001 to April 2006. Pathology reports were screened for those cases that were negative for estrogen and progesterone receptors and HER-2/neu (triple negative). A total of 16 cases $(16 / 98,16 \%)$ fulfilled this screening criterion. The histologic slides, including the case estrogen/progesterone/HER-2/neu immunostains were reviewed and immunohistologic stains were performed for cytokeratins 14,17 and 5/6, vimentin, EGFR, c-kit, smooth muscle actin and p63. Immunohistochemistry was performed on the Benchmark XT, and developed with iView DAB (Ventana, Tuscon, AZ); the details are summarized in Table 1.

Immunohistochemistry slides were semiquantitated as follows: EGFR, HER-2/neu and c-Kit were graded according to the accepted grading scheme for Her2/neu, as $0,1+, 2+, 3+$. Briefly, a score of zero has no immunostaining, $1+$ has $10 \%$ of cells or less with weak incomplete cell membrane immunostaining, $2+$ has at least $10 \%$ of cells with complete, weak to moderate cell membrane immunostaining and $3+$ has at least $10 \%$ of cells with strong complete membrane immunostaining. HER-2/neu was considered to be positive if immunostaining was $3+$ or if a $2+$ result showed gene amplification by FISH. Any immunostaining $(1+$ to $3+)$ was considered to be a positive result for c-Kit and EGFR.

Hormone receptors were reviewed and accepted as negative if $100 \%$ of cells lacked nuclear immunostaining for hormone receptor.

The cytokeratins, vimentin, p63 and smooth muscle actin were graded as 0 (negative), R (rare single cells stain), $1+(5-30 \%$ cells stain $), 2+$ $(31-60 \%$ cells stain) and $3+(>60 \%$ of cells stain).

Tumor size was recorded for the patient group and lymph nodes were examined.

Table 1 Antibodies, sources and conditions

\begin{tabular}{lllll}
\hline Antibody to & Source & Clone & Conditions & Titer \\
\hline EGFR & Ventana & 3C6 & Protease 3 & Predil \\
CK5/6 & Cell Marque & D5 and 16B4 & CC1 & Predil \\
SMA & Cell Marque & 1A4 & CC1 & Predil \\
CK 17 & Dako & E3 & CC1 & $1: 20$ \\
CK 14 & Cell Marque & LL002 & CC1 & $1: 60$ \\
P63 & Dako & 4A4 & CC1 & $1: 400$ \\
Her2/neu & Novocastra & CB11 & CC1 & $1: 200$ \\
ER & Ventana & 6F11 & CC1 & Predil \\
PR & Ventana & 1A6 & CC1 & Predil \\
Vimentin & Ventana & V9 & CC1 & Predil \\
& & & & \\
\hline
\end{tabular}

${ }^{a}$ All antibodies were used on the Benchmark XT (Ventana, Tuscon, AZ) and developed with iView DAB.

\section{Results}

The mean number of histologic slides was 17 slides per case, excluding lymph nodes. There were at least three sections of tumor for each case. The tumor sizes ranged from 0.2 to $3.1 \mathrm{~cm}$, with a mean of $1.9 \mathrm{~cm}$ and a median of $2.5 \mathrm{~cm}$. In all, 11 patients had axillary lymph node dissections and five had sentinel lymph node biopsies. The number of lymph nodes ranged from 2 to 19 , and five patients had sentinel biopsies only. Only one patient in the entire study group had a solitary $2 \mathrm{~mm}$ (sentinel) lymph node metastasis.

Of the 16 cases, 13 had morphologic components of ductal carcinoma in situ (DCIS), all of which were located on the immediate periphery of the invasive component. The DCIS was verified with the p63, CK14 and smooth muscle actin immunostains, which documented the presence of myoepithelial cells in the ducts containing DCIS. The DCIS morphologic types were solid (11 cases, eight with comedonecrosis), flat 'clinging' (one case) or micropapillary (one case) mixed with solid. All were nuclear grade 3 and had an intense lymphoid inflammatory infiltrate, and never comprised more than $5-10 \%$ of the neoplasm.

All cases with DCIS exhibited an immunostaining profile analogous to the invasive component (Figures 1 and 2).

The immunostaining results are summarized in Table 2. Cytokeratin 5/6 showed positive immunostaining in $15 / 16$ cases, with a $3+$ result in $10 / 16$, and a $2+$ result in three cases. Cytokeratin 17 was $3+$ positive in $9 / 16$ cases, and $2+$ in $2 / 16$ cases. Cytokeratin 14 was $3+$ positive in only $5 / 16$ cases and weakly positive in the remainder. Two cases in which HER-2 immunostain was reported as $2+$ were both negative by FISH. Vimentin was positive in all cases, strongly in 12/16 cases. EGFR was positive in $15 / 16$ cases, $3+$ in $6 / 16,2+$ in $4 / 16$ and $1+$ in $5 / 16$ cases.

C-Kit was positive in 6/16 cases, smooth muscle actin in 7/16 cases and p63 showed sporadic staining of tumor cells in the majority of cases. Immunoreactivity with p63 and smooth muscle actin was seen only in a minority of cells in a patchy fashion in almost all cases.

\section{Discussion}

Traditional histologic classification of breast carcinoma along with histologic grading provide meaningful biologic information. In addition, estrogen receptor and HER-2 status have provided us with more accurate prognostic and predictive tools. In recent years, gene expression profiling data have further refined the subtypes of breast carcinomas. ${ }^{1-4,12}$

Among the five subgroups (luminal A, luminal B, normal breast-like, ERB2 + and basal-like) of breast carcinoma identified by gene expression profiling, 

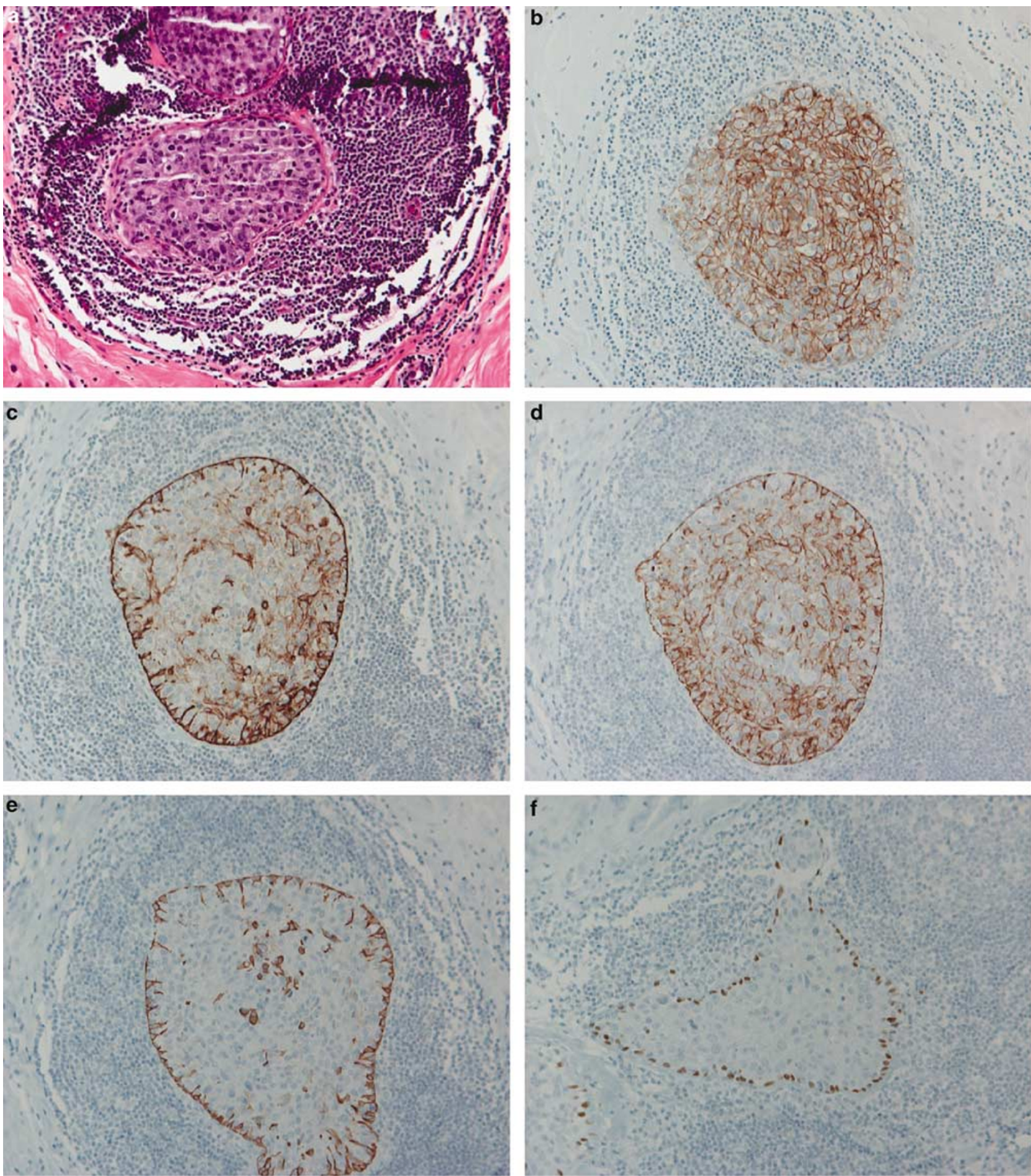

Figure 1 (a) Solid DCIS, high nuclear grade with lymphoid infiltrate, hematoxylin/eosin. (b) Strong EGFR membrane staining, DAB. (c) CK17 has strong cytoplasmic and myoepithelial cell staining, DAB. (d) CK5/6 shows strong diffuse tumor cell staining along with myoepithelial cells, DAB. (e) CK14 stains few tumor cells and the myoepithelial cells, DAB. (f) P63 immunostains only the myoepithelial cells in this case.

most attention has been given to basal-like carcinoma. The possible interest is due to the fact that these tumors were not well recognized before the expression profiling, but in retrospect they seem to have a relatively specific immunohistologic profile. It appears that some reports have previously described the morphologic aspects of basal-like carcinomas before the gene expression profiling experiments. ${ }^{13-16}$

It has been known for several years that myoepithelial and luminal epithelial cells have different 

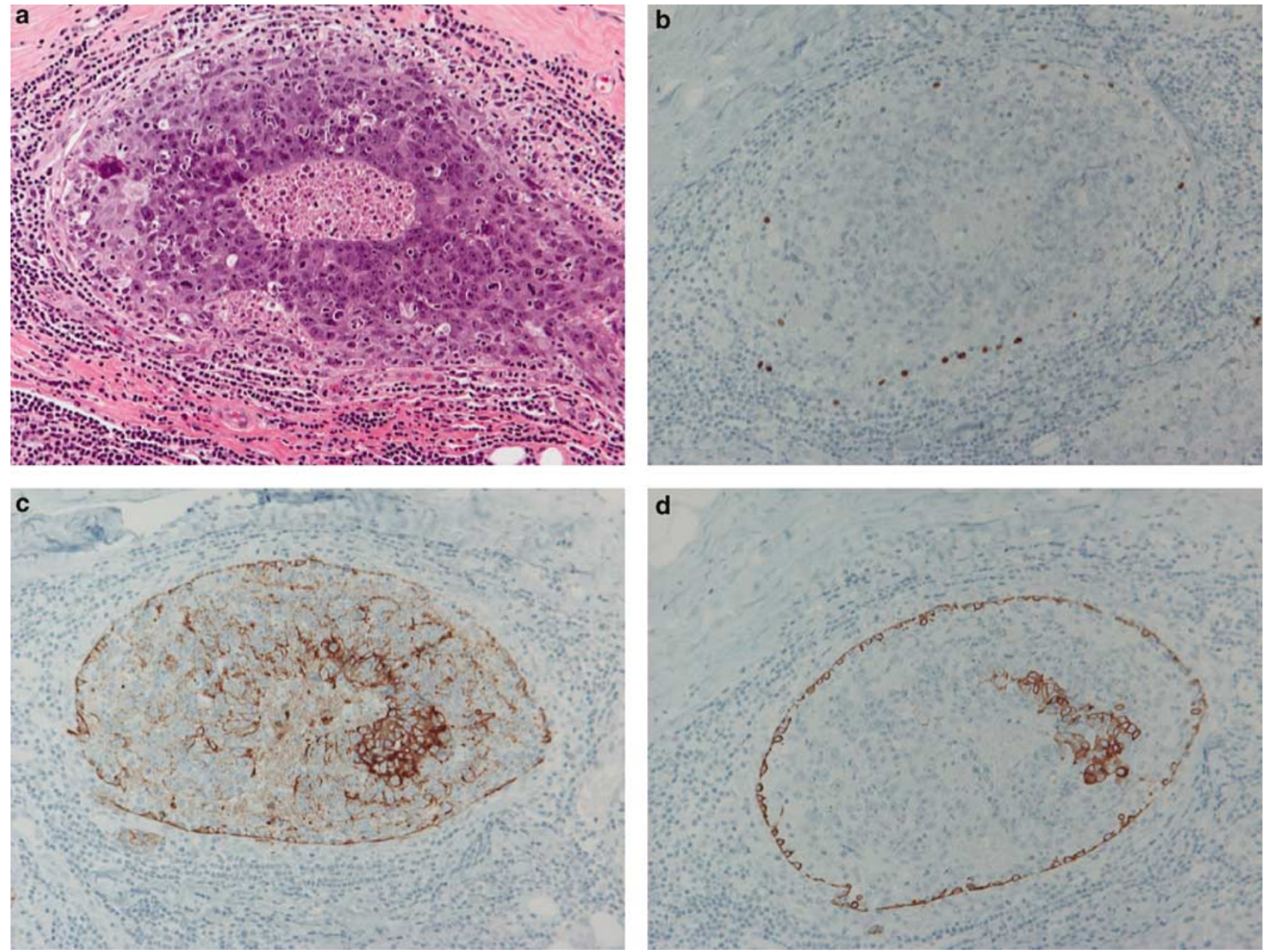

Figure 2 (a) Solid DCIS with comedonecrosis and high nuclear grade, hematoxylin/eosin. (b) P63 stains only the myoepithelial cells, DAB. (c) CK5/6 shows strong tumor cell staining along with myoepithelial cells, DAB. (d) CK 14 shows focal tumor cell staining along with myoepithelial cells, DAB.

Table 2 Immunostaining results of basal type of breast carcinoma

Case Ck14 Ck17 Ck5/6 EGFR VIM HER2 cKit P63 SMA

\begin{tabular}{llllllllll}
\hline 1 & $\mathrm{R}$ & $3^{\mathrm{a}}$ & $2^{\mathrm{a}}$ & $2^{\mathrm{a}}$ & $3^{\mathrm{a}}$ & 0 & 0 & $\mathrm{R}$ & $1^{\mathrm{a}}$ \\
2 & $3^{\mathrm{a}}$ & $3^{\mathrm{a}}$ & $3^{\mathrm{a}}$ & $3^{\mathrm{a}}$ & $3^{\mathrm{a}}$ & 0 & 0 & $2^{\mathrm{a}}$ & $2^{\mathrm{a}}$ \\
3 & $3^{\mathrm{a}}$ & $3^{\mathrm{a}}$ & $3^{\mathrm{a}}$ & $1^{\mathrm{a}}$ & $1^{\mathrm{a}}$ & 0 & $3^{\mathrm{a}}$ & 0 & $3^{\mathrm{a}}$ \\
4 & $3^{\mathrm{a}}$ & $3^{\mathrm{a}}$ & $3^{\mathrm{a}}$ & $1^{\mathrm{a}}$ & $3^{\mathrm{a}}$ & 0 & 0 & $\mathrm{R}$ & 0 \\
5 & $3^{\mathrm{a}}$ & $3^{\mathrm{a}}$ & $3^{\mathrm{a}}$ & $1^{\mathrm{a}}$ & $1^{\mathrm{a}}$ & $1^{\mathrm{a}}$ & $1^{\mathrm{a}}$ & $\mathrm{R}$ & 0 \\
6 & $1^{\mathrm{a}}$ & $2^{\mathrm{a}}$ & $3^{\mathrm{a}}$ & $3^{\mathrm{a}}$ & $1^{\mathrm{a}}$ & 0 & $1^{\mathrm{a}}$ & $\mathrm{R}$ & 0 \\
7 & $3^{\mathrm{a}}$ & $3^{\mathrm{a}}$ & $3^{\mathrm{a}}$ & $2^{\mathrm{a}}$ & $3^{\mathrm{a}}$ & 0 & $1^{\mathrm{a}}$ & $\mathrm{R}$ & 0 \\
8 & $1^{\mathrm{a}}$ & $3^{\mathrm{a}}$ & $3^{\mathrm{a}}$ & $1^{\mathrm{a}}$ & $3^{\mathrm{a}}$ & 0 & $1^{\mathrm{a}}$ & $1^{\mathrm{a}}$ & 0 \\
9 & $1^{\mathrm{a}}$ & $2^{\mathrm{a}}$ & $3^{\mathrm{a}}$ & $3^{\mathrm{a}}$ & $3^{\mathrm{a}}$ & $2^{\mathrm{a}, \mathrm{b}}$ & 0 & $1^{\mathrm{a}}$ & 0 \\
10 & $1^{\mathrm{a}}$ & $1^{\mathrm{a}}$ & $\mathrm{R}$ & $2^{\mathrm{a}}$ & $3^{\mathrm{a}}$ & $2^{\mathrm{a}, \mathrm{b}}$ & 0 & $1^{\mathrm{a}}$ & $1^{\mathrm{a}}$ \\
11 & $1^{\mathrm{a}}$ & $3^{\mathrm{a}}$ & $2^{\mathrm{a}}$ & $2^{\mathrm{a}}$ & $1^{\mathrm{a}}$ & $1^{\mathrm{a}}$ & 0 & $1^{\mathrm{a}}$ & 0 \\
12 & $1^{\mathrm{a}}$ & $1^{\mathrm{a}}$ & $3^{\mathrm{a}}$ & $1^{\mathrm{a}}$ & $3^{\mathrm{a}}$ & 0 & $1^{\mathrm{a}}$ & $\mathrm{R}$ & $2^{\mathrm{a}}$ \\
13 & $1^{\mathrm{a}}$ & $1^{\mathrm{a}}$ & $2^{\mathrm{a}}$ & $3^{\mathrm{a}}$ & $3^{\mathrm{a}}$ & $1^{\mathrm{a}}$ & $1^{\mathrm{a}}$ & 0 & $1^{\mathrm{a}}$ \\
14 & $1^{\mathrm{a}}$ & $1^{\mathrm{a}}$ & $1^{\mathrm{a}}$ & 0 & $3^{\mathrm{a}}$ & 0 & 0 & 0 & 0 \\
15 & $1^{\mathrm{a}}$ & $\mathrm{R}$ & $1^{\mathrm{a}}$ & $3^{\mathrm{a}}$ & $3^{\mathrm{a}}$ & 0 & 0 & $1^{\mathrm{a}}$ & 0 \\
16 & $1^{\mathrm{a}}$ & $3^{\mathrm{a}}$ & $3^{\mathrm{a}}$ & $3^{\mathrm{a}}$ & $3^{\mathrm{a}}$ & 0 & 0 & $3^{\mathrm{a}}$ & $3^{\mathrm{a}}$ \\
\end{tabular}

${ }^{\mathrm{a}}$ Estrogen and progesterone receptors were both negative in $100 \%$ of cells in all cases.

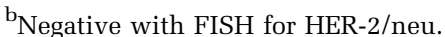

immunoprofiles. ${ }^{17}$ The basal-like carcinomas are socalled because they express 'basal-type cytokeratins' CK5 and CK17, that is, cytokeratins expressed by basal/myoepithelial cells of the normal breast. In the original gene expression profiling experiment, the basal-like carcinomas had the worst clinical outcome. $^{1,2}$ Recently, Livasy et $a l^{5}$ reported that basal-like carcinomas are histologically poorly differentiated, have high nuclear grade, show geographic necrosis and stromal lymphocytic response. These tumors are 'triple negative' (negative for ER, PR and HER-2) and express vimentin, EGFR and CK5/6 by immunohistochemistry. Myoepithelial markers such as SMA, CD10 and p63 are expressed infrequently. A subset of basal-like carcinoma may also include a few metaplastic carcinomas. Unfortunately, Livasy et al did not comment on the presence or absence of in situ carcinomas or other precursor lesions in their 23 cases.

In order to validate the characteristic immunohistologic findings and to identify any precursor lesion of basal-like carcinomas, we searched our pathology database for breast carcinomas in women less than 37 years of age. This was performed simply to increase the yield of high-grade, ER-negative breast carcinomas, as these are more common in the younger age group. 
The majority of BRCA-1-associated tumors have the basal phenotype, ${ }^{8,18,19}$ but the $B R C A-1$ status was not available for any of our cases.

Our morphologic review, and immunohistochemical stains revealed almost identical findings as reported by Livasy et al. ${ }^{5}$ The tumors were all high grade (Nottingham score 9/9), with variable geographic necrosis, moderate to good microscopic circumscription and significant lymphoid inflammatory cell infiltrates The tumors were also positive for EGFR (94\%) and vimentin (100\%). Staining for other sensitive and specific myoepithelial markers (SMA, C-kit, p63) was seen in less than $50 \%$ of cases, which indicates that these tumors demonstrate a partial myoepithelial phenotype. Studies on fetal and infant breasts have shown that cells at the tips of the lobular buds and terminal end buds have a characteristic cytoskeletal protein profile, and may have the capacity to generate both basal and luminal cells. ${ }^{20}$ It is likely that basal-like carcinomas originate from such 'stem cells' and therefore demonstrate expression of immunomarkers of both luminal and myoepithelial/basal types.

Our most significant and previously unreported finding was the presence of ductal carcinoma in situ (DCIS) along with the invasive basal-like carcinomas in $13 / 16(81 \%)$ of cases. Most often, the in situ component was only focally present, on the immediate periphery of the neoplasm, and in no case more than $10 \%$ of the entire tumor volume. The presence of DCIS was confirmed with multiple immunostains. Ductal carcinoma in situ showed a variety of morphologic patterns, including the solid, flat and micropapillary types. However, all in situ carcinomas were of high nuclear grade and the majority showed comedo-type necrosis. Interestingly, the in situ carcinomas demonstrated the same immunophenotype as the invasive carcinomas and hence, provide evidence for the in situ precursor lesion. Atypical ductal hyperplasia was not identified in any of our cases. The absence of atypical hyperplasia and presence of small quantities of in situ carcinoma suggest that these tumors grow very rapidly, become invasive and in many instances obliterate the in situ carcinoma from which they arise. In a recent study by Bryan et $a{ }^{21}{ }^{21}$ ductal carcinoma in situ with basal-like phenotype was described. Unfortunately, the study consisted of only pure DCIS without an associated invasive carcinoma, and therefore the authors speculated that invasive basal-like carcinomas might have a precursor DCIS lesion. In our study, we provide evidence that invasive basal-like carcinomas do indeed have a precursor lesion in the form of DCIS.

Another finding of interest was the presence of intense inflammatory lymphoid infiltrate around the DCIS. The pattern of inflammatory infiltrate resembled the pattern seen with regressive changes in a high-grade DCIS. It is conceivable that some high-grade DCIS with regressive changes likely represent 'basal-like DCIS'; however, additional studied are required to prove this assumption.

In summary, basal-like carcinomas express markers of basal/myoepithelial cells that are found in normal breast tissue. Our study confirms and extends the previously reported findings of the basal-like carcinoma immunophenotype. ${ }^{5-8,10}$ In addition, we demonstrate the presence of in situ carcinoma as the likely precursor lesion for invasive basal-like carcinoma.

We agree with Nielson et $a l^{10}$ that a panel consisting of the triple negative ER/PR/HER-2, along with CK5/6 and EGFR, will identify basal-like carcinomas with great precision. Immunostaining with cytokeratins 14 and 17 may also be helpful. The reporting of basal-like carcinoma on routine histopathologic examination may be helpful for future clinical outcome studies. The identification of high-grade DCIS with comedonecrosis and lymphoid inflammatory infiltrates on core biopsy or resection specimens should raise the diagnostic possibility of a basal-type DCIS, which could be confirmed by immunohistology.

\section{References}

1 Sorlie T, Tibshirani R, Parker J, et al. Repeated observation of breast tumor subtypes in independent gene expression data sets. Proc Natl Acad Sci USA 2003;100:8418-8423.

2 Sorlie T, Perou CM, Tibshirani R, et al. Gene expression patterns of breast carcinomas distinguish tumor subclasses with clinical implications. Proc Natl Acad Sci USA 2001;98:10869-10874.

3 Perou CM, Sorlie T, Eisen MB, et al. Molecular portraits of human breast tumours. Nature 2000;406:747-752.

4 Perou CM, Jeffrey SS, van de Rijn M, et al. Distinctive gene expression patterns in human mammary epithelial cells and breast cancers. Proc Natl Acad Sci USA 1999;96:9212-9217.

5 Livasy CA, Karaca G, Nanda R, et al. Phenotypic evaluation of the basal-like subtype of invasive breast carcinoma. Mod Pathol 2006;19:264-271.

6 Ribeiro-Silva A, Ramalho LN, Garcia SB, et al. p63 correlates with both BRCA1 and cytokeratin 5 in invasive breast carcinomas: further evidence for the pathogenesis of the basal phenotype of breast cancer. Histopathology 2005;47:458-466.

7 Matos I, Dufloth R, Alvarenga M, et al. p63, cytokeratin 5, and P-cadherin: three molecular markers to distinguish basal phenotype in breast carcinomas. Virchows Arch 2005;447:688-694.

8 Laakso M, Loman N, Borg A, et al. Cytokeratin 5/14positive breast cancer: true basal phenotype confined to BRCA1 tumors. Mod Pathol 2005;18:1321-1328.

9 Gusterson BA, Ross DT, Heath VJ, et al. Basal cytokeratins and their relationship to the cellular origin and functional classification of breast cancer. Breast Cancer Res 2005;7:143-148.

10 Nielsen TO, Hsu FD, Jensen $\mathrm{K}$, et al. Immunohistochemical and clinical characterization of the basal-like subtype of invasive breast carcinoma. Clin Cancer Res 2004;10:5367-5374. 
11 Abd El-Rehim DM, Pinder SE, Paish CE, et al. Expression of luminal and basal cytokeratins in human breast carcinoma. J Pathol 2004;203:661-671.

12 Sotiriou C, Neo SY, McShane LM, et al. Breast cancer classification and prognosis based on gene expression profiles from a population-based study. Proc Natl Acad Sci USA 2003;100:10393-10398.

13 Domagala W, Wozniak L, Lasota J, et al. Vimentin is preferentially expressed in high-grade ductal and medullary, but not in lobular breast carcinomas. Am J Pathol 1990;137:1059-1064.

14 Domagala W, Lasota J, Dukowicz A, et al. Vimentin expression appears to be associated with poor prognosis in node-negative ductal NOS breast carcinomas. Am J Pathol 1990;137:1299-1304.

15 Domagala W, Lasota J, Bartkowiak J, et al. Vimentin is preferentially expressed in human breast carcinomas with low estrogen receptor and high Ki-67 growth fraction. Am J Pathol 1990;136:219-227.

16 Cattoretti G, Andreola S, Clemente C, et al. Vimentin and p53 expression on epidermal growth factor receptor-positive, oestrogen receptor-negative breast carcinomas. Br J Cancer 1988;57:353-357.

17 Nagle RB, Bocker W, Davis JR, et al. Characterization of breast carcinomas by two monoclonal antibodies distinguishing myoepithelial from luminal epithelial cells. J Histochem Cytochem 1986;34:869-881.

18 Lakhani SR, Reis-Filho JS, Fulford L, et al. Prediction of BRCA1 status in patients with breast cancer using estrogen receptor and basal phenotype. Clin Cancer Res 2005;11:5175-5180.

19 Foulkes WD, Stefansson IM, Chappuis PO, et al. Germline BRCA1 mutations and a basal epithelial phenotype in breast cancer. J Natl Cancer Inst 2003;95: 1482-1485.

20 Anbazhagan R, Osin PP, Bartkova J, et al. The development of epithelial phenotypes in the human fetal and infant breast. J Pathol 1998;184:197-206.

21 Bryan BB, Schnitt SJ, Collins LC. Ductal carcinoma in situ with basal-like phenotype: a possible precursor to invasive basal-like breast cancer. Mod Pathol 2006; 19:617-621. 